\title{
A FETI-DP Formulation for Two-dimensional Stokes Problem on Nonmatching Grids *
}

\author{
Hyea Hyun Kim ${ }^{1}$ and Chang-Ock Lee ${ }^{2}$ \\ 1 Division of Applied Mathematics, KAIST, Daejeon, 305-701, Korea \\ (mashy@amath.kaist.ac.kr) \\ 2 Division of Applied Mathematics, KAIST (colee@amath.kaist.ac.kr)
}

\begin{abstract}
Summary. We consider a FETI-DP formulation of the Stokes problem with mortar methods. To solve the Stokes problem correctly and efficiently, redundant continuity constraints are introduced. Lagrange multipliers corresponding to the redundant constraints are treated as primal variables in the FETI-DP formulation. We propose a preconditioner for the FETI-DP operator and show that the condition number of the preconditioned FETI-DP operator is bounded by $C \max _{i=1, \cdots, N}\left\{\left(1+\log \left(H_{i} / h_{i}\right)\right)^{2}\right\}$, where $H_{i}$ and $h_{i}$ are the subdomain size and the mesh size, respectively, and $C$ is a constant independent of $H_{i}$ and $h_{i}$.
\end{abstract}

\section{Introduction}

Recently, FETI-DP methods, which were originally developed by Farhat et al. [2001], have been applied to nonconforming discretizations( Dryja and Widlund [2002, 2003], Kim and Lee [2002]). Nonconforming discretizations are important for multiphysics simulations, contact-impact problems, the generation of meshes and partitions aligned with jumps in diffusion coefficients, $h p$-adaptive methods, and special discretizations in the neighborhood of singularities. For the elliptic problems in 2D, Dryja and Widlund [2002] showed that the Dirichlet preconditioner gives the condition number bound $C(1+\log (H / h))^{4}$, where $H$ and $h$ denote the subdomain size and the mesh size, respectively. Further, Dryja and Widlund [2003] proposed a different preconditioner which is similar to the one in Klawonn and Widlund [2001], and proved the condition number bound $C(1+\log (H / h))^{2}$ with a restriction that the mesh sizes on the nonmortar side and the mortar side are comparable. For the same problem, Kim and Lee [2002] formulated a FETI-DP operator in a different way from Dryja and Widlund [2002, 2003] and proposed a Neumann-Dirichlet preconditioner, which gives the condition number bound $C(1+\log (H / h))^{2}$ without the restriction on mesh sizes between neighboring subdomains. For the elliptic problems with heterogeneous coefficients, they

^ This work was partially supported by KOSEF R01-2000-00008. 
obtained the same condition number bound which does not depend on the coefficients.

In this paper, we extend the result in Kim and Lee [2002] to the Stokes problem. We use the inf-sup stable $P_{1}(h)-P_{0}(2 h)$ finite elements in each subdomain. For the optimality of the approximation under nonmatching discretizations, we impose mortar matching conditions on the velocity functions using the standard Lagrange multiplier space introduced in Bernardi et al. [1994].

\section{FETI-DP formulation}

Let $\Omega$ be a bounded polygonal domain in $\mathbb{R}^{2}$. We assume that $\Omega$ is partitioned into nonoverlapping bounded polygonal subdomains $\left\{\Omega_{i}\right\}_{i=1}^{N}$ and the partition is geometrically conforming. Let $H_{D}^{1}\left(\Omega_{i}\right)$ be a space of functions in $H^{1}\left(\Omega_{i}\right)$ with zero traces on $\partial \Omega_{i} \cap \partial \Omega, L_{0}^{2}\left(\Omega_{i}\right)$ be a space of functions in $L^{2}\left(\Omega_{i}\right)$ with zero average and $\Pi^{0}$ be a space of functions in $L_{0}^{2}(\Omega)$ which are constants in each subdomain. Then, we consider the following variational form of Stokes' problem: Find $\left(\mathbf{u}, p_{I}, p^{0}\right) \in \prod_{i=1}^{N}\left[H_{D}^{1}\left(\Omega_{i}\right)\right]^{2} \times \prod_{i=1}^{N} L_{0}^{2}\left(\Omega_{i}\right) \times \Pi^{0}$ such that

$$
\begin{aligned}
\sum_{i=1}^{N}(\nabla \mathbf{u}, \nabla \mathbf{v})_{\Omega_{i}}-\sum_{i=1}^{N} & \left(p_{I}+p^{0}, \nabla \cdot \mathbf{v}\right)_{\Omega_{i}}=\sum_{i=1}^{N}(\mathbf{f}, \mathbf{v})_{\Omega_{i}} \quad \forall \mathbf{v} \in \prod_{i=1}^{N}\left[H_{D}^{1}\left(\Omega_{i}\right)\right]^{2} \\
-\sum_{i=1}^{N}\left(\nabla \cdot \mathbf{u}, q_{I}\right)_{\Omega_{i}} & =0 \quad \forall q_{I} \in \prod_{i=1}^{N} L_{0}^{2}\left(\Omega_{i}\right) \\
-\sum_{i=1}^{N}\left(\nabla \cdot \mathbf{u}, q^{0}\right)_{\Omega_{i}} & =0 \quad \forall q^{0} \in \Pi^{0}
\end{aligned}
$$

and the velocity $\mathbf{u}$ is continuous across the subdomain interfaces $\Gamma=$ $\bigcup_{i, j=1}^{N}\left(\partial \Omega_{i} \cap \partial \Omega_{j}\right)$. Here, $(\cdot, \cdot)_{\Omega_{i}}$ denotes the inner product in $\left[L^{2}\left(\Omega_{i}\right)\right]^{n}$ for $n=1,2$.

We associate $\Omega_{i}$ with quasi-uniform triangulations $\Omega_{i}^{h_{i}}$ and $\Omega_{i}^{2 h_{i}}$. Then we consider the inf-sup stable $P_{1}\left(h_{i}\right)-P_{0}\left(2 h_{i}\right)$ finite elements and denote them by $X_{i}$ and $Q_{i}$, respectively. In addition, $Q_{i}^{0}$ is defined as a subspace of $Q_{i}$ with zero average on $\Omega_{i}$. Let $W_{i}:=X_{i}$ for all $i=1, \cdots, N$. To get a FETI-DP formulation, we define the following spaces:

$$
\begin{aligned}
& X:=\left\{\mathbf{v} \in \prod_{i=1}^{N} X_{i}: \mathbf{v} \text { is continuous at subdomain corners }\right\}, \\
& W=\left\{\mathbf{w} \in \prod_{i=1}^{N} W_{i}: \mathbf{w} \text { is continuous at subdomain corners }\right\},
\end{aligned}
$$




$$
Q:=\prod_{i=1}^{N} Q_{i}^{0}
$$

In this paper, we will use the same notation for a finite element function and the vector of nodal values of that function. The same applies to the notations $W_{i}, X, W$, etc. For $\mathbf{v}_{i} \in X_{i}$, we write

$$
\mathbf{v}_{i}^{t}=\left(\left(\mathbf{v}_{I}^{i}\right)^{t}\left(\mathbf{v}_{r}^{i}\right)^{t}\left(\mathbf{v}_{c}^{i}\right)^{t}\right),
$$

where the symbol $I, r$ and $c$ represent the d.o.f.(degrees of freedom) at the interior nodes, nodes on edges and corners, respectively. Since $\mathbf{v} \in X$ is continuous at subdomain corners, there exists a vector $\mathbf{v}_{c}$ such that $\mathbf{v}_{c}^{i}=L_{c}^{i} \mathbf{v}_{c}$ for all $i=1, \cdots, N$ with a restriction map $L_{c}^{i}$. The vector $\mathbf{v}_{c}$ has the d.o.f. corresponding to the union of subdomain corners. Let

$$
\mathbf{v}_{I}^{t}=\left(\left(\mathbf{v}_{I}^{1}\right)^{t} \cdots\left(\mathbf{v}_{I}^{N}\right)^{t}\right), \quad \mathbf{v}_{r}^{t}=\left(\left(\mathbf{v}_{r}^{1}\right)^{t} \cdots\left(\mathbf{v}_{r}^{N}\right)^{t}\right) .
$$

We define the spaces $X_{I}, W_{r}$ and $W_{c}$ which consist of vectors $\mathbf{v}_{I}, \mathbf{v}_{r}$ and $\mathbf{v}_{c}$, respectively. For $\mathbf{w} \in W$, we define $\mathbf{w}_{r} \in W_{r}$ and $\mathbf{w}_{c} \in W_{c}$ similarly to $\mathbf{v}_{r}$ and $\mathbf{v}_{c}$.

On each $\Gamma_{i j}\left(=\partial \Omega_{i} \cap \partial \Omega_{j}\right)$, we determine mortar and nonmortar sides and define

$$
\begin{aligned}
m_{i} & :=\left\{j:\left.\Omega_{i}^{h}\right|_{\Gamma_{i j}} \text { is the nonmortar side of } \Gamma_{i j}\right\}, \\
s_{i} & :=\left\{j:\left.\Omega_{i}^{h}\right|_{\Gamma_{i j}} \text { is the mortar side of } \Gamma_{i j}\right\} .
\end{aligned}
$$

We consider the standard Lagrange multiplier space $M_{i j}$ and let

$$
M:=\prod_{i=1}^{N} \prod_{j \in m_{i}} M_{i j} .
$$

Then the following mortar matching conditions are imposed on $v \in X$ :

$$
\int_{\Gamma_{i j}}\left(\mathbf{v}_{i}-\mathbf{v}_{j}\right) \cdot \boldsymbol{\lambda}_{i j} d s=0 \quad \forall \boldsymbol{\lambda}_{i j} \in M_{i j}, \forall i=1, \cdots, N, \forall j \in m_{i} .
$$

Now, we rewrite (2) into a matrix form. Let $B_{i}^{i j}$ be a matrix with entries

$$
\left(B_{i}^{i j}\right)_{l k}= \pm \int_{\Gamma_{i j}} \boldsymbol{\psi}_{l} \cdot \boldsymbol{\phi}_{k} d s \quad \forall l=1, \cdots, L, \forall k=1, \cdots, K,
$$

where $\left\{\boldsymbol{\psi}_{l}\right\}_{l=1}^{L}$ is basis for $M_{i j}$ and $\left\{\phi_{k}\right\}_{k=1}^{K}$ is nodal basis for $\left.W_{i}\right|_{\Gamma_{i j}}$. In (3), the +sign is chosen if $\left.\Omega_{i}\right|_{\Gamma_{i j}}$ is a nonmortar side, otherwise the -sign is chosen. Let $E_{i j}: M_{i j} \rightarrow M$ be an extension operator by zero and $R_{i j}^{l}:\left.W_{l} \rightarrow W_{l}\right|_{\Gamma_{i j}}$ for $l=i, j$ be a restriction operator and $B_{i}=\sum_{j \in m_{i} \cup s_{i}} E_{i j} B_{i}^{i j} R_{i j}^{i}$. Then (2) is written into 


$$
B \mathbf{w}=\mathbf{0},
$$

where $B=\left(B_{1} \cdots B_{N}\right)$ and $\mathbf{w}=\left(\mathbf{w}_{1}^{t} \cdots \mathbf{w}_{N}^{t}\right)^{t}$ with $\mathbf{w}_{i}=\left.\mathbf{v}_{i}\right|_{\partial \Omega_{i}}$. Let $B_{i, r}$ and $B_{i, c}$ be matrices that consist of the columns of $B_{i}$ corresponding to the d.o.f. on edges and corners, respectively. Then (4) is written into

$$
B_{r} \mathbf{w}_{r}+B_{c} \mathbf{w}_{c}=\mathbf{0}
$$

where $B_{r}=\left(B_{1, r} \cdots B_{N, r}\right)$ and $B_{c}=\sum_{i=1}^{N} B_{i, c} L_{c}^{i}$.

Borrowing the idea of $\mathrm{Li}$ [2001], we add the following redundant continuity constraints to the coarse problem

$$
\int_{\Gamma_{i j}}\left(\mathbf{v}_{i}-\mathbf{v}_{j}\right) d s=\mathbf{0} \quad \forall i=1, \cdots, N, \forall j \in m_{i} .
$$

and rewrite (5) as

$$
R^{t}\left(B_{r} \mathbf{w}_{r}+B_{c} \mathbf{w}_{c}\right)=\mathbf{0}
$$

with a suitable matrix $R$. Let $S$ be the Lagrange multiplier space corresponding to the constraints (6).

Then, the following is induced from the Galerkin approximation to (1): Find $\left(\mathbf{u}_{I}, p_{I}, \mathbf{u}_{r}, \mathbf{u}_{c}, p^{0}, \boldsymbol{\mu}, \boldsymbol{\lambda}\right) \in X_{I} \times Q \times W_{r} \times W_{c} \times \Pi^{0} \times S \times M$ such that

$$
\left(\begin{array}{ccccccc}
A_{I I} & G_{I I} & A_{I r} & A_{I c} & \mathbf{0} & \mathbf{0} & \mathbf{0} \\
G_{I I}^{t} & \mathbf{0} & G_{r I}^{t} & G_{c I}^{t} & \mathbf{0} & \mathbf{0} & \mathbf{0} \\
A_{r I} & G_{r I} & A_{r r} & A_{r c} & G_{r 0} & B_{r}^{t} R & B_{r}^{t} \\
A_{c I} & G_{c I} & A_{c r} & A_{c c} & G_{c 0} & B_{c}^{t} R & B_{c}^{t} \\
\mathbf{0} & \mathbf{0} & G_{r 0}^{t} & G_{c 0}^{t} & \mathbf{0} & \mathbf{0} & \mathbf{0} \\
\mathbf{0} & \mathbf{0} & R^{t} B_{r} & R^{t} B_{c} & \mathbf{0} & \mathbf{0} & \mathbf{0} \\
\mathbf{0} & \mathbf{0} & B_{r} & B_{c} & \mathbf{0} & \mathbf{0} & \mathbf{0}
\end{array}\right)\left(\begin{array}{c}
\mathbf{u}_{I} \\
p_{I} \\
\mathbf{u}_{r} \\
\mathbf{u}_{c} \\
p^{0} \\
\boldsymbol{\mu} \\
\boldsymbol{\lambda}
\end{array}\right)=\left(\begin{array}{c}
\mathbf{f}_{I} \\
\mathbf{0} \\
\mathbf{f}_{r} \\
\mathbf{f}_{c} \\
\mathbf{0} \\
\mathbf{0} \\
\mathbf{0}
\end{array}\right) .
$$

Let

$$
\mathbf{z}_{r}^{t}=\left(\mathbf{u}_{I}^{t} p_{I}^{t} \mathbf{u}_{r}^{t}\right), \quad \mathbf{z}_{c}^{t}=\left(\mathbf{u}_{c}^{t}\left(p^{0}\right)^{t} \boldsymbol{\mu}^{t}\right) .
$$

In the FETI-DP formulation, we regard $\mathbf{z}_{c}$ as a primal variable. After eliminating $\mathbf{z}_{r}$ and $\mathbf{z}_{c}$, we obtain the following equation for $\boldsymbol{\lambda}$

$$
F_{D P} \boldsymbol{\lambda}=\mathbf{d}
$$

and call $F_{D P}$ a FETI-DP operator.

\section{Preconditioner}

We define $S_{i}$ as the discrete Schur complement operator of the Stokes problem in $\Omega_{i}$ obtained by eliminating interior velocity and pressure unknowns. Let

$$
S:=\operatorname{diag}\left(S_{1}, \cdots, S_{N}\right)
$$


and it can be seen easily that $S$ is s.p.d. on $W$. Hence, we define a norm for $\mathbf{w} \in W$ as

$$
\|\mathbf{w}\|_{W}^{2}:=\sum_{i=1}^{N}<S_{i} \mathbf{w}_{i}, \mathbf{w}_{i}>.
$$

Let

$$
W^{0}=\prod_{i=1}^{N} \prod_{j \in m_{i}} W_{i j}^{0},
$$

where $W_{i j}^{0}$ consists of functions in $\left.W_{i}\right|_{\Gamma_{i j}}$ with zero value at the end points of $\Gamma_{i j}$. For a function $\mathbf{w}_{i j} \in W_{i j}^{0}$ with $j \in m_{i}$, let $\widetilde{\mathbf{w}}_{i j} \in W_{i}$ be the zero extension of $\mathbf{w}_{i j}$. Using this, we define the zero extension $\widetilde{\mathbf{w}} \in W$ of $\mathbf{w} \in W^{0}$ by

$$
\widetilde{\mathbf{w}}=\left(\widetilde{\mathbf{w}}_{1}, \cdots, \widetilde{\mathbf{w}}_{N}\right) \text { with } \widetilde{\mathbf{w}}_{i}=\sum_{j \in m_{i}} \widetilde{\mathbf{w}}_{i j}
$$

and a norm on $W^{0}$ by

$$
\|\mathbf{w}\|_{W^{0}}:=\|\widetilde{\mathbf{w}}\|_{W} .
$$

We introduce the following subspaces with the norms induced from the spaces $W$ and $W^{0}$ :

$$
\begin{aligned}
W_{R} & :=\left\{\mathbf{w} \in W: R^{t}\left(B_{r} \mathbf{w}_{r}+B_{c} \mathbf{w}_{c}\right)=\mathbf{0}\right\}, \\
W_{R, G} & :=\left\{\mathbf{w} \in W_{R}: G_{r 0}^{t} \mathbf{w}_{r}+G_{c 0}^{t} \mathbf{w}_{c}=\mathbf{0}\right\}, \\
W_{R}^{0} & :=\left\{\mathbf{w} \in W^{0}: \tilde{\mathbf{w}} \in W_{R}\right\} .
\end{aligned}
$$

Let us define

$$
M_{R}=\left\{\boldsymbol{\lambda} \in M: R^{t} \boldsymbol{\lambda}=\mathbf{0}\right\}
$$

and a dual norm for $\boldsymbol{\lambda} \in M_{R}$ by

$$
\|\boldsymbol{\lambda}\|_{M_{R}}^{2}:=\max _{\mathbf{w} \in W_{R}^{0} \backslash\{0\}} \frac{\left\langle\boldsymbol{\lambda}, \mathbf{w}>_{m}^{2}\right.}{\|\mathbf{w}\|_{W^{0}}^{2}},
$$

where $\left\langle\boldsymbol{\lambda}, \mathbf{w}>_{m}=\sum_{i=1}^{N} \sum_{j \in m_{i}} \int_{\Gamma_{i j}} \boldsymbol{\lambda}_{i j} \cdot \mathbf{w}_{i j} d s\right.$ is a duality pairing. From this dual norm, we can find an operator $\widehat{F}_{D P}$ which gives

$$
<\widehat{F}_{D P} \boldsymbol{\lambda}, \boldsymbol{\lambda}>=\|\boldsymbol{\lambda}\|_{M_{R}}^{2}
$$

and propose $\widehat{F}_{D P}^{-1}$ as a preconditioner for the FETI-DP operator in (7). To give a matrix form of $\widehat{F}_{D P}^{-1}$, we define $R_{i j}: W^{0} \rightarrow W_{i j}^{0}$ as a restriction operator and $E_{i j}^{i}: W_{i j}^{0} \rightarrow W_{i}$ as an extension operator by zero. Let $\widehat{B}_{i}^{i j}$ be a matrix obtained from $B_{i}^{i j}$ after deleting the columns corresponding to the end points of $\Gamma_{i j}$. Since, we restrict $\boldsymbol{\lambda} \in M_{R}$ and $\mathbf{w} \in W_{R}^{0}$, we need $l^{2}$-orthogonal projections

$$
P_{W_{R}^{0}}^{i j}:\left.\left.W^{0}\right|_{\Gamma_{i j}} \rightarrow W_{R}^{0}\right|_{\Gamma_{i j}}, \quad P_{M_{R}}^{i j}:\left.\left.M\right|_{\Gamma_{i j}} \rightarrow M_{R}\right|_{\Gamma_{i j}} .
$$


Let $\widehat{B}_{i j}=P_{M_{R}}^{i j} \widehat{B}_{i}^{i j} P_{W_{R}^{0}}^{i j}$ and $\widehat{B}_{i}=\sum_{j \in m_{i}} E_{i j}^{i} \widehat{B}_{i j}^{-1} R_{i j}$. Then, we obtain

$$
\widehat{F}_{D P}^{-1}=\sum_{i=1}^{N} \widehat{B}_{i}^{t} S_{i} \widehat{B}_{i}
$$

Thus, the computation of $\widehat{F}_{D P}^{-1} \boldsymbol{\lambda}$ can be done parallely in each subdomain.

\section{Condition number estimation}

In this section, we only state lemmas that are used to analyze the condition number bound without proofs. In the following, $C$ is a generic constant independent of $h_{i}$ and $H_{i}$.

Lemma 1. For $\boldsymbol{\lambda} \in M_{R}$, we have

$$
<F_{D P} \boldsymbol{\lambda}, \boldsymbol{\lambda}>=\max _{\mathbf{w} \in W_{R, G} \backslash\{0\}} \frac{<B \mathbf{w}, \boldsymbol{\lambda}>^{2}}{\|\mathbf{w}\|_{W}^{2}} .
$$

Lemma 2. For $\boldsymbol{\lambda} \in M_{R}$, we have

$$
\max _{\mathbf{w} \in W_{R, G} \backslash\{0\}} \frac{<B \mathbf{w}, \boldsymbol{\lambda}>^{2}}{\|\mathbf{w}\|_{W}^{2}} \geq\|\boldsymbol{\lambda}\|_{M_{R}}^{2} .
$$

Let us define a notation $|\cdot|_{S_{i}}:=<S_{i} \cdot, \cdot>^{1 / 2}$. Then the following lemma can be found in Bramble and Pasciak [1990].

Lemma 3. For $\mathbf{w}_{i} \in W_{i}$, we have

$$
C_{1} \beta\left|\mathbf{w}_{i}\right|_{S_{i}} \leq\left|\mathbf{w}_{i}\right|_{1 / 2, \partial \Omega_{i}} \leq C_{2}\left|\mathbf{w}_{i}\right|_{S_{i}},
$$

where $\beta$ is the inf-sup constant for the finite elements of subdomain $\Omega_{i}$ and the constants $C_{1}$ and $C_{2}$ are independent of $h_{i}$ and $H_{i}$.

We also have the following result which is derived from Lemma 5.1 in Mandel and Tezaur [2001].

Lemma 4. For $\mathbf{w} \in W$, we have

$\left\|\mathbf{w}_{i}-\mathbf{w}_{j}\right\|_{H_{00}^{1 / 2}\left(\Gamma_{i j}\right)}^{2} \leq C \max _{l \in\{i, j\}}\left\{\left(1+\log \frac{H_{l}}{h_{l}}\right)^{2}\right\}\left(\left|\mathbf{w}_{i}\right|_{1 / 2, \partial \Omega_{i}}^{2}+\left|\mathbf{w}_{j}\right|_{1 / 2, \partial \Omega_{j}}^{2}\right)$.

From Lemma 3, Lemma 4 and the continuity of mortar projection in $H_{00}^{1 / 2}\left(\Gamma_{i j}\right)$, we have

Lemma 5. For $\boldsymbol{\lambda} \in M_{R}$,

$$
\max _{\mathbf{w} \in W_{R, G} \backslash\{0\}} \frac{<B \mathbf{w}, \boldsymbol{\lambda}>^{2}}{\|\mathbf{w}\|_{W}^{2}} \leq C \max _{i=1, \cdots, N}\left\{\left(1+\log \frac{H_{i}}{h_{i}}\right)^{2}\right\}\|\boldsymbol{\lambda}\|_{M_{R}}^{2} .
$$


From Lemma 1, Lemma 2, Lemma 5 and (8), we obtain the following condition number bound:

Theorem 1.

$$
\kappa\left(\widehat{F}_{D P}^{-1} F_{D P}\right) \leq C \max _{i=1, \cdots, N}\left\{\left(1+\log \frac{H_{i}}{h_{i}}\right)^{2}\right\} .
$$

\section{Numerical Results}

Let $\Omega=[0,1] \times[0,1] \subset \mathbb{R}^{2}$ and consider Stokes problem with an exact solution

$$
\mathbf{u}=\left(\begin{array}{c}
\sin ^{3}(\pi x) \sin ^{2}(\pi y) \cos (\pi y) \\
-\sin ^{2}(\pi x) \sin ^{3}(\pi y) \cos (\pi x)
\end{array}\right) \text { and } p=x^{2}-y^{2}
$$

Let $N$ denote the number of subdomains. We only consider the uniform partition of $\Omega$. The notation $N=4 \times 4$ means that $\Omega$ is partitioned into $4 \times 4$ square subdomains. Let $n$ denote the number of nodes on subdomain edges including end points, which is associated with $\Omega_{i}^{h_{i}}$, a triangulation for velocity functions. We solve the FETI-DP operator with and without preconditioner varying $N$ and $n$ under nonmatching discretizations. Those cases are denoted by PFETI-DP and FETI-DP, respectively. The CG(Conjugate Gradient) iteration is stopped when the relative residual is less than $10^{-6}$.

In Tables 1 and 2, the number of CG iterations and the corresponding condition number are shown varying $N$ and $n$. From Table 1 , we observe that PFETI-DP performs well and the condition numbers seem to behave $\log ^{2}$ growth as $n$ increases. In Table 2 , as $N$ increases with $n=5$ or $n=9$, the CG iteration becomes stable for both cases with and without preconditioner. Hence, we can see that the developed preconditioner gives the condition number bound as confirmed in theory.

Table 1. CG iterations(condition number) when $N=4 \times 4$

\begin{tabular}{l|ll}
\hline $\mathrm{n}$ & FETI-DP & PFETI-DP \\
\hline 5 & $16(8.35)$ & $12(3.75)$ \\
9 & $50(1.15 \mathrm{e}+2)$ & $15(5.79)$ \\
17 & $86(5.01 \mathrm{e}+2)$ & $17(7.93)$ \\
33 & $119(1.31 \mathrm{e}+3)$ & $20(9.88)$ \\
65 & $153(3.29 \mathrm{e}+3)$ & $22(1.20 \mathrm{e}+1)$ \\
\hline
\end{tabular}


Table 2. CG iterations(condition number) when $n=5,9$

\begin{tabular}{l|ll|ll}
\hline \multirow{2}{*}{$N$} & $\begin{array}{l}n=5 \\
\text { FETI-DP }\end{array}$ & $\begin{array}{l}n=9 \\
\end{array}$ & & \\
\hline $4 \times 4$ & $16(8.35)$ & $12(3.75)$ & $50(1.15 \mathrm{e}+2)$ & $15(5.79)$ \\
$8 \times 8$ & $16(9.18)$ & $12(3.68)$ & $53(1.19 \mathrm{e}+2)$ & $15(6.21)$ \\
$16 \times 16$ & $16(9.57)$ & $11(3.42)$ & $57(1.34 \mathrm{e}+2)$ & $16(6.27)$ \\
$32 \times 32$ & $16(10.88)$ & $12(3.78)$ & $56(1.25 \mathrm{e}+2)$ & $16(6.24)$ \\
\hline
\end{tabular}

\section{References}

C. Bernardi, Y. Maday, and A. T. Patera. A new nonconforming approach to domain decomposition: the mortar element method. In Nonlinear partial differential equations and their applications. Collège de France Seminar, Vol. XI (Paris, 1989-1991), volume 299 of Pitman Res. Notes Math. Ser., pages 13-51. Longman Sci. Tech., Harlow, 1994.

J. H. Bramble and J. E. Pasciak. A domain decomposition technique for Stokes problems. Appl. Numer. Math., 6(4):251-261, 1990.

M. Dryja and O. B. Widlund. A FETI-DP method for a mortar discretization of elliptic problems. In Recent developments in domain decomposition methods (Zürich, 2001), volume 23 of Lect. Notes Comput. Sci. Eng., pages 41-52. Springer, Berlin, 2002.

M. Dryja and O. B. Widlund. A generalized FETI-DP method for a mortar discretization of elliptic problems. In Domain decomposition methods in Science and Engineering (Cocoyoc, Mexico, 2002), pages 27-38. UNAM, Mexico City, 2003.

C. Farhat, M. Lesoinne, P. LeTallec, K. Pierson, and D. Rixen. FETI-DP: a dual-primal unified FETI method. I. A faster alternative to the two-level FETI method. Internat. J. Numer. Methods Engrg., 50(7):1523-1544, 2001.

H. H. Kim and C.-O. Lee. A FETI-DP preconditioner for elliptic problems on nonmatching grids. In KAIST DAM Research Report 02-9. KAIST, 2002.

A. Klawonn and O. B. Widlund. FETI and Neumann-Neumann iterative substructuring methods: connections and new results. Comm. Pure Appl. Math., 54(1):57-90, 2001.

J. Li. A dual-primal FETI method for incompressible Stokes equations. In Technical Report 816. Department of Computer Science, Courant Institute, New York Unversity, 2001.

J. Mandel and R. Tezaur. On the convergence of a dual-primal substructuring method. Numer. Math., 88(3):543-558, 2001. 